\title{
Using computerized workflow simulations to assess the feasibility of whole slide imaging full adoption in a high-volume histology laboratory
}

\author{
David S. McClintock*, Roy E. Lee and John R. Gilbertson \\ Department of Pathology, Massachusetts General Hospital, Boston, MA, USA
}

\begin{abstract}
Background: Whole slide Imaging (WSI) has been touted by many as the future of pathology, with estimates of full adoption occurring sometime in the next 5 to 15 years. While WSI devices have become increasingly capable since their inception, there has been little consideration of how WSI will be implemented and subsequently affect the workflow of high-volume histology laboratories.

Methods: Histology workflow process data was collected from a high-volume histology laboratory (Massachusetts General Hospital) and a process model developed using business process management software. Computerized workflow simulations were performed and total histology process time evaluated under a number of different WSI conditions.

Results: Total histology process time increased approximately 10-fold to 20 -fold over baseline with the presence of one WSI robot in the histology workflow. Depending on the specifications of the WSI robot, anywhere from 9 to 14 WSI robots were required within the histology workflow to minimize the effects of WSI.

Conclusions: Placing a WSI robot into the current workflow of a high-volume histology laboratory with the intent of full adoption is not feasible. Implementing WSI without making significant changes to the current workflow of the histology laboratory would prove to be both disruptive and costly to surgical pathology.
\end{abstract}

Keywords: Whole slide imaging, virtual microscopy, workflow, histology, business analysis software, computer simulations, process modeling, BPMN

\section{Introduction}

Since the introduction of whole slide imaging (WSI) in 1999, the technology surrounding this field has advanced to the point where placing a WSI device, or robot, into the traditional pathology workflow does not seem as inconceivable as it once was. In its early years, whole slide imaging was plagued by technological barriers that made it difficult to implement within pathology laboratories, such as slow scan times at

${ }^{*}$ Corresponding author: David S. McClintock, MD, Department of Pathology, Warren Building 225, Massachusetts General Hospital, Boston, MA 02114, USA. Tel.: +1 773405 9450; Fax: +1 617643 4470; E-mail: dsmcclin@gmail.com. clinically relevant slide magnifications, the inability to deliver multiple reliable focal planes (z-axis planes), extremely large file sizes, and no support for the Digital Imaging and Communications in Medicine (DICOM) standard for medical image sharing [1,2]. These barriers were significant enough that WSI was largely relegated to the "post-diagnostic" phase of pathology, with glass slides scanned only after the pathologist had finished with them and rendered a diagnosis.

Over the past few years, however, advances in both general computing (processor, memory, and graphics updates; larger storage devices; faster networking speeds, etc.) and WSI technology (faster scanning times, more reliable automation and handling of slides, more robust image viewers, etc.) have reduced the 
impact of past technical barriers [3], paving the way for the possibility of placing a WSI robot within the "pre-diagnostic" phase of pathology. Logistically, however, the fact remains that a virtual microscopic image must begin with the glass slide. Therefore, a whole slide image begins not at the point of scanning, but instead with the processing, cutting and direct staining of tissue within the clinical histology laboratory. Incorporating WSI into the pre-diagnostic phase of pathology necessitates that WSI robots be physically placed within the clinical histology laboratory and integrated within the histology workflow [4].

While innovation in whole slide imaging has largely been focused on reducing and eliminating the technical barriers prohibiting widespread clinical use, little has been done to investigate the logistical barriers surrounding its use within histology laboratories and ultimately, its effect on surgical pathology workflow. In this study, our aim was to investigate the effects WSI has on clinical histology laboratory workflow in a more quantitative manner than before [4] through the use of business process analysis software. Specifically, we wished to assess the current feasibility for the full adoption of whole slide imaging in pathology (scanning every slide that comes through the clinical histology laboratory on a daily basis) by answering the following questions: How does adding a WSI robot to a high-volume clinical histology laboratory affect the turn-around-time (TAT) of slides being delivered to pathologists? How many WSI robots will be needed to maintain the workflow of the clinical histology laboratory as it is today? How fast will slides need to be scanned in order to maintain the current workflow?

\section{Methods}

Outside of medicine, business process analysis software solutions exist to help businesses, and even entire industries, break down the complexity of their workflows, reduce redundant processes, and improve efficiency. Within healthcare, a review of the literature reveals that application of these common business tools, while not unprecedented, is not widespread. Further, within medicine, there are only a handful of publications noting the use of business analysis software [e.g. 5-8], and even fewer in pathology [9, 10].

In so far as pathology is a division of the healthcare enterprise, it also can be considered from an industry standpoint as being comprised of repeatable, automated to semi-automated workflows and processes, all serving to deliver the pathology report as the final end product. In anatomic pathology, this is definitely true of the clinical histology laboratory, which, depending on the size of the pathology service, can produce hundreds to thousands of slides per day. By documenting the workflow and applying business process analytical software to the clinical histology laboratory, one can begin to model and then simulate the effects that whole slide imaging will have on this workflow.

At Massachusetts General Hospital (MGH), workflow process data for the clinical histology laboratory was collected regarding: 1) process steps in the workflow, 2) available equipment and personnel resources, and 3) estimated volume of daily assets (blocks and slides). Process steps in the workflow were initially documented using business process modeling notation (BPMN, www.bpmn.org) in Microsoft Visio 2007 (Microsoft, visio.microsoft.com) (Fig. 1). From this initial process map, a baseline process model for the clinical histology laboratory was developed using the business analysis software suite $\mathrm{iGrafx}^{\circledR} 2011$ (Corel, www.igrafx.com) per the method below.

Briefly, a process map of the desired workflow is created (typically using BPMN) and data then assigned to each of the process tasks, or activities within the map. Swimlanes (horizontal rows) in the process map denote different "departments" or "divisions" within the workflow where activities are performed. Depending on the defined activity, for example if the activity is the start of a process (rounded rectangle), an activity in the process (rectangle), or a decision within the process (diamond), there are specific data elements that can be assigned to further characterize the behavior of that activity. A process model is created once all of the desired data elements have been assigned to the activities within a process map. Computerized simulations can then be run and data generated by introducing transactions into the model at one or more activities. By changing the data elements assigned to an activity, or by removing or introducing activities (e.g. adding a WSI robot to the baseline histology workflow), new models can be created and new data generated from which comparisons can be made.

Data to complete the baseline clinical histology laboratory process model at MGH was gathered through two main methods: 1) direct observation where available (equipment, personnel, and specific histology task durations), and 2) best estimates from the laboratory supervisors and technical director (volumes of daily assets, specific histology task durations). Specific data elements collected for this model are described in 


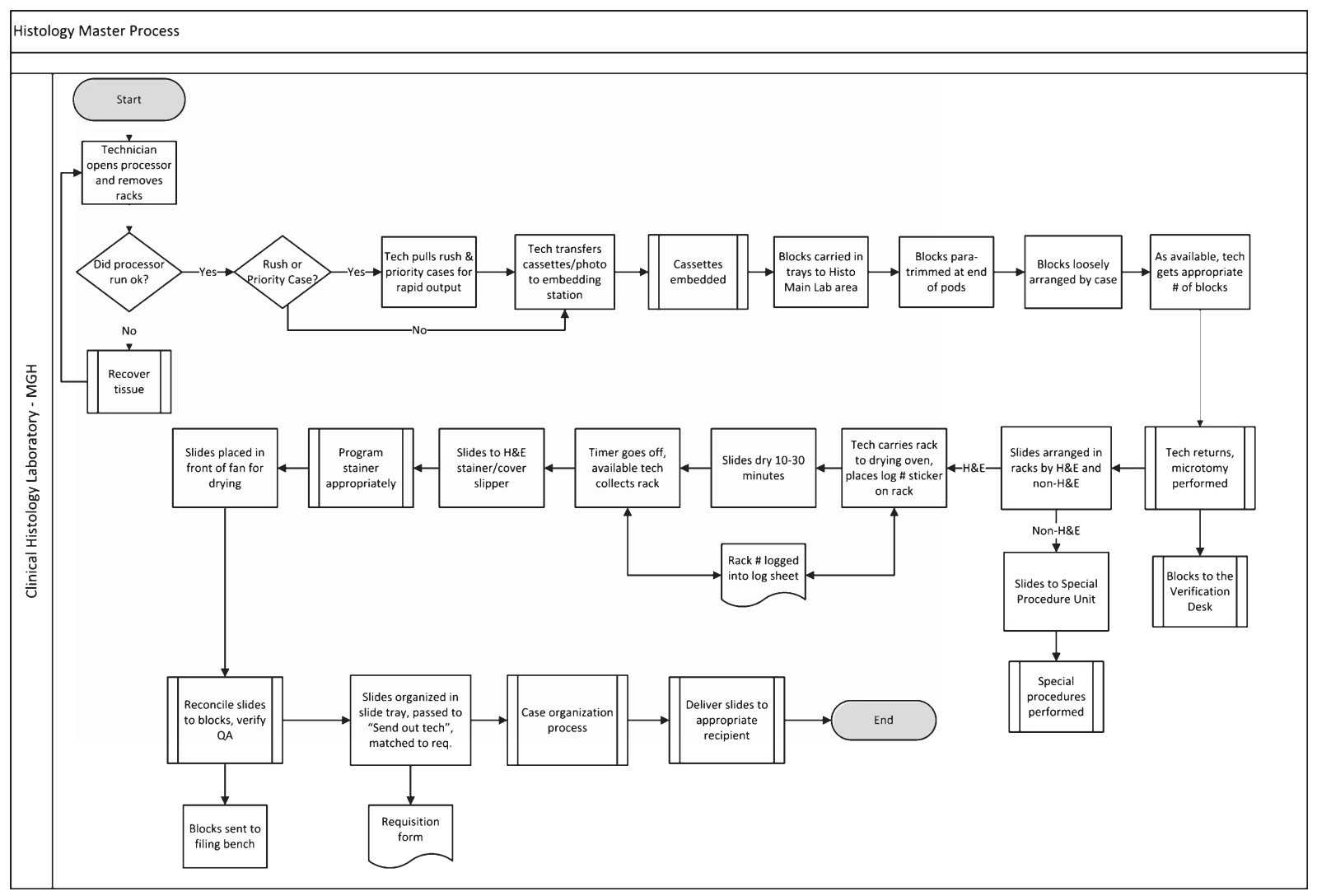

Fig. 1. Histology Master Process, Microsoft Visio Diagram, using BPMN notation.

Table 1 and the completed baseline clinical histology process model is shown in Fig. 2.

Computerized simulations were run in iGraf $\mathrm{x}^{\circledR} 2011$ using blocks and slides as the transactions and the volumes generated based on an average routine day in the MGH clinical histology laboratory (Fig. 3). Simulation parameters, including inputs and assumptions, are described in Table 2.

\section{Results}

\subsection{Effects of a single WSI robot on turn-around-time within the clinical histology laboratory}

To investigate the effects a single whole slide imaging robot on clinical histology workflow, the baseline process model was compared to one with a WSI robot placed between the end of slide creation but before slides were distributed to pathologists (Fig. 4). Simulations were run on this "WSI robot" model using four different slide scanning time ranges, based on both the scan times claimed by a variety of WSI vendors and user experience of the authors (60-180 sec, 90-180 sec, 60-240 sec, and 120-240 sec) and laboratory TAT was measured. As seen in Fig. 5, placing a single WSI robot within the clinical histology laboratory workflow resulted in significant increases in TAT, adding a minimum 107.95 hours ( 6.17 work days, 10.4-fold increase over baseline) to a maximum 208.95 hours (11.94 work days, 20.1-fold increase over baseline) to finish the daily histology work for the scan times chosen.

\subsection{Whole slide imaging robots and maintaining the current clinical histology workflow}

Given the marked increase in turn-around-time by placing only a single WSI robot within the 
Table 1

Data elements used for process modeling

\begin{tabular}{|c|c|}
\hline Data element type & Description \\
\hline Generators & $\begin{array}{l}\text { Introduce transactions (assets) into the process model - for the histology laboratory, transactions are defined as } \\
\text { blocks and slides }\end{array}$ \\
\hline Event generator & Introduces a bolus of transactions into the process at a given time (e.g. 200 blocks from a 6 AM processor run) \\
\hline Activity & Performs an action on a transaction via a defined set of instructions and assigned resources \\
\hline Task inputs & $\begin{array}{l}\text { Describes a collection behavior for transactions as they enter an activity (e.g., batching slides into racks, joining } \\
\text { slides to create a case, etc.) }\end{array}$ \\
\hline Task duration & $\begin{array}{l}\text { Amount of time needed to complete the activity - can be a constant value, a distributed range of time, or an } \\
\text { expression/formula }\end{array}$ \\
\hline Task on completion & $\begin{array}{l}\text { Specifies a task to be performed on a transaction upon completion of the activity (e.g., duplicate transactions, } \\
\text { discard transactions, etc.) }\end{array}$ \\
\hline Task outputs & $\begin{array}{l}\text { Specifies the path a transaction can take following activity completion (e.g. normal path through process vs. } \\
\text { exceptions) }\end{array}$ \\
\hline Resources & $\begin{array}{l}\text { Every activity within the process must have a resource assigned to it in order to perform an action, or work, on an } \\
\text { asset }\end{array}$ \\
\hline Workers & $\begin{array}{l}\text { Personnel who are needed to perform an action on a transaction (e.g., microtomy technician, embedding } \\
\text { technician, etc.) }\end{array}$ \\
\hline Equipment & $\begin{array}{l}\text { Equipment needed to perform an action or that is needed for a worker to perform an action on a transaction (e.g., } \\
\text { microtomy station, slide stainer, WSI robot, etc.) }\end{array}$ \\
\hline
\end{tabular}

Basic Histology

Model

Blocks $=1200$

Slides $=2400$

No WSI Robot

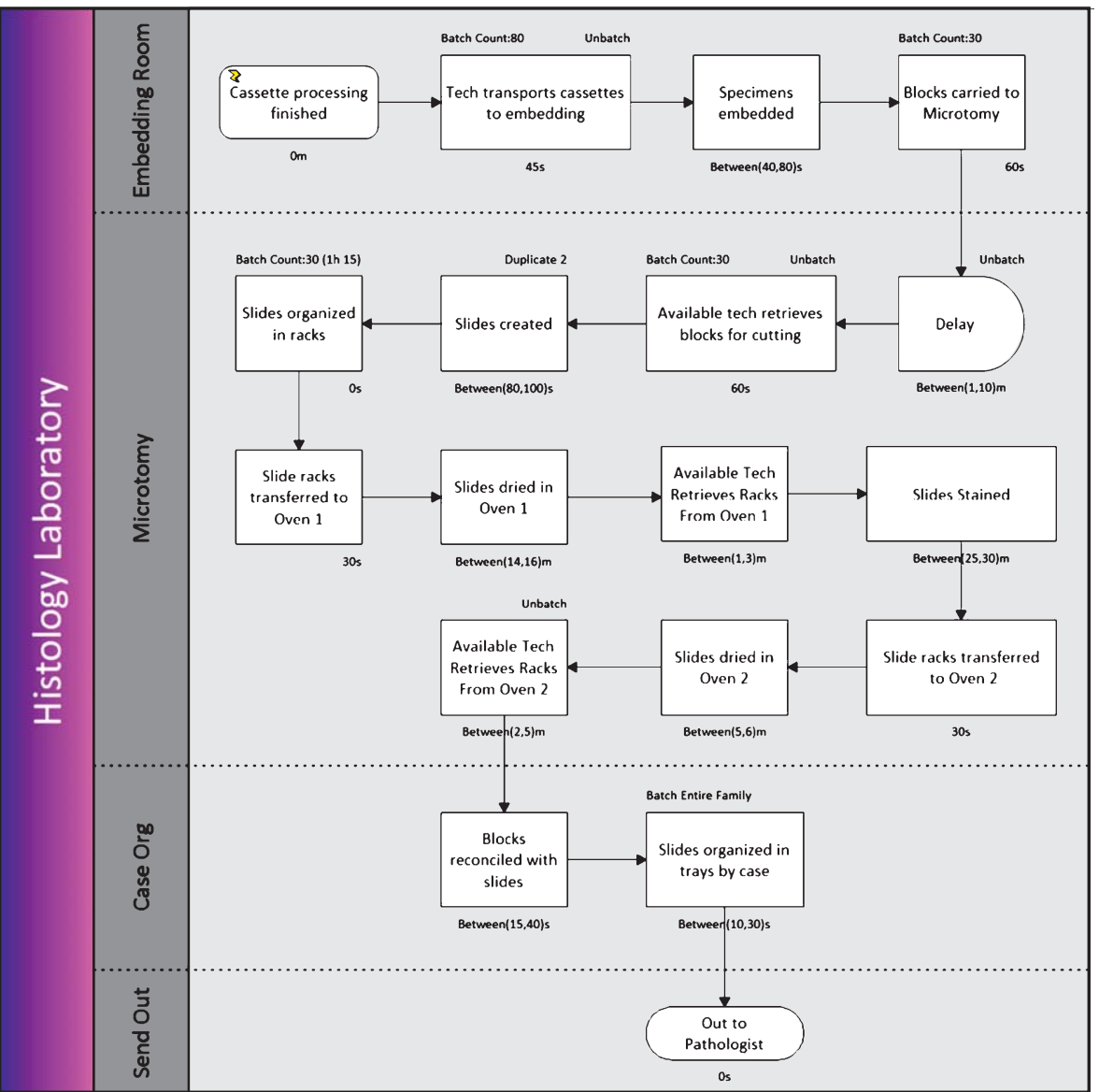

Fig. 2. Baseline clinical histology laboratory process model in iGrafx ${ }^{\circledR} 2011$. 


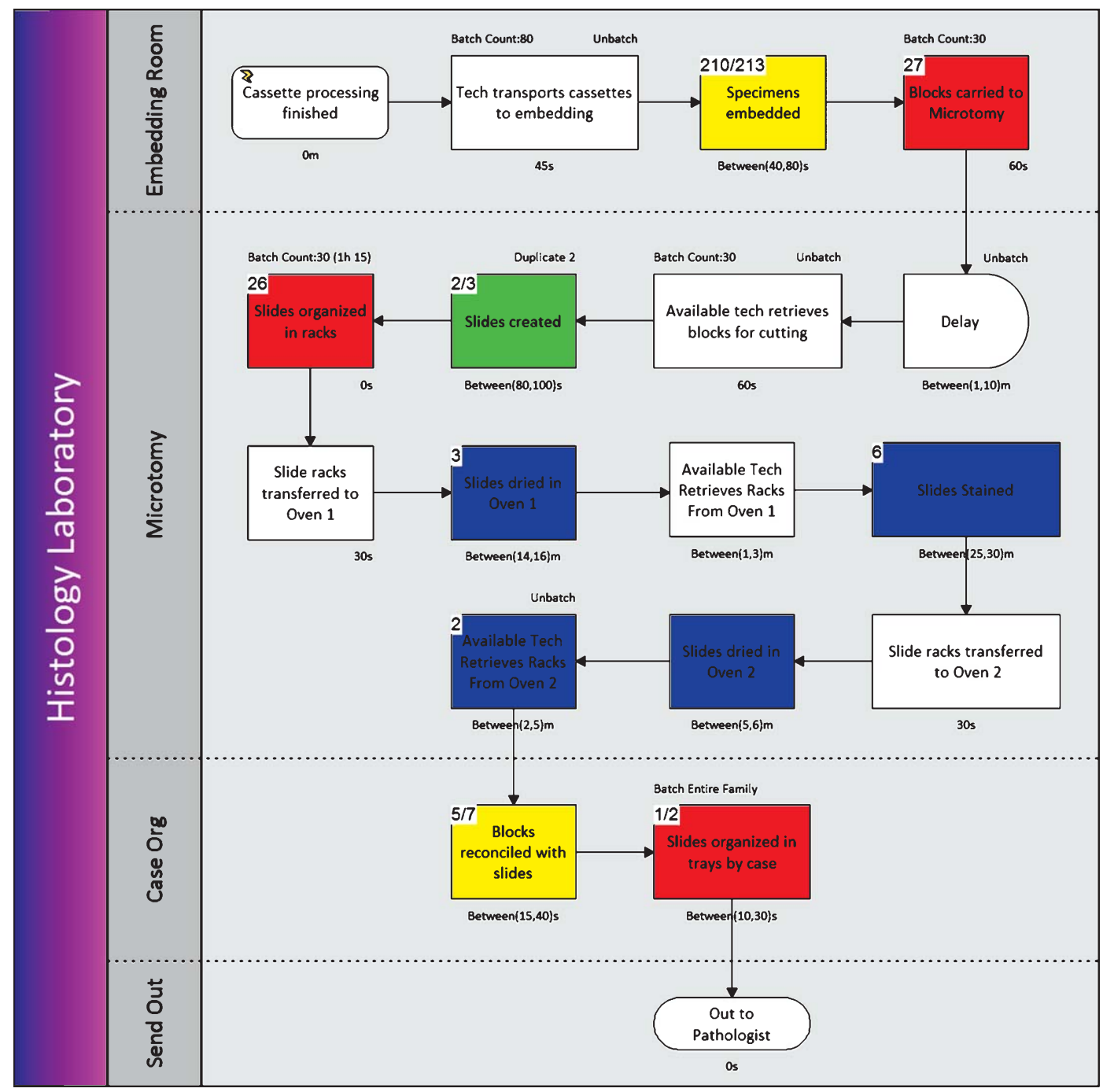

Fig. 3. Running simulations in iGraf $\mathrm{x}^{\circledR} 2011$ (trace mode) with the following color representations: Green, transactions (blocks or slides) moving through the process; Blue, work being done on a transaction or group of transactions; Red, task blocked until task rules are met (batch or join activity), Yellow, transaction is waiting for a resource and cannot continue until one is free; White, activity inactive (no transactions).

histology workflow, the next logical step was to see how many WSI robots would be needed to minimize their effects on the clinical histology laboratory. Using the previously described "WSI robot" process model, an additional WSI robot was added as a resource for the model and a simulation was run. This sequence was repeated for each of the chosen scan time ranges, adding additional WSI robots until no further decrease in the total TAT of the histology laboratory workflow was noted. As seen in Fig. 6, a minimum of $9(60-180 \mathrm{sec})$ to a maximum of $14(120-240 \mathrm{sec})$ additional WSI robots were needed to completely minimize the effects of WSI on the histology workflow.

\subsection{Optimizing WSI robot scan times in the current clinical histology workflow}

Instead of adding additional WSI robots to the workflow, a different approach towards full adoption of WSI could be to continue down the line of technical innovation and further decrease the scan time per glass slide. 
Table 2

Simulation parameters used for the baseline clinical histology laboratory and the WSI robot process models

\begin{tabular}{ll}
\hline Simulation parameters & Description \\
\hline Schedule & Reflects the routine daily working schedule of the clinical histology laboratory at Massachusetts General \\
Hospital (MGH) & Monday thru Friday, work week starts on Monday \\
Work week & $2: 30$ AM to $8: 00$ PM \\
Rours & Reflects the average routine daily volume of the clinical histology laboratory at MGH \\
Inputs & 1200 blocks introduced into the model from 4 processor batches: \\
Blocks & $2: 30$ AM, 480 blocks \\
& $5: 00$ AM, 280 blocks \\
& $5: 30$ AM, 240 blocks \\
& $6: 00$ AM, 200 blocks \\
& Process model was created for "routine" work only - Same Day/Rush/ Priority blocks were not introduced in this \\
model & 2400 slides, 2 slides per block created (duplication of transactions upon completion of "Slides created" activity \\
in model) & Direct survey of resources, reflects the current workers and equipment present in the clinical histology laboratory \\
at MGH & Worked over the entire day with no breaks, assumption was made that all workers were available when needed \\
Resources & Quantities and behaviors of instruments taken directly from the lab \\
Not accounted for at this time, all activities were assumed to have been completed with no errors
\end{tabular}

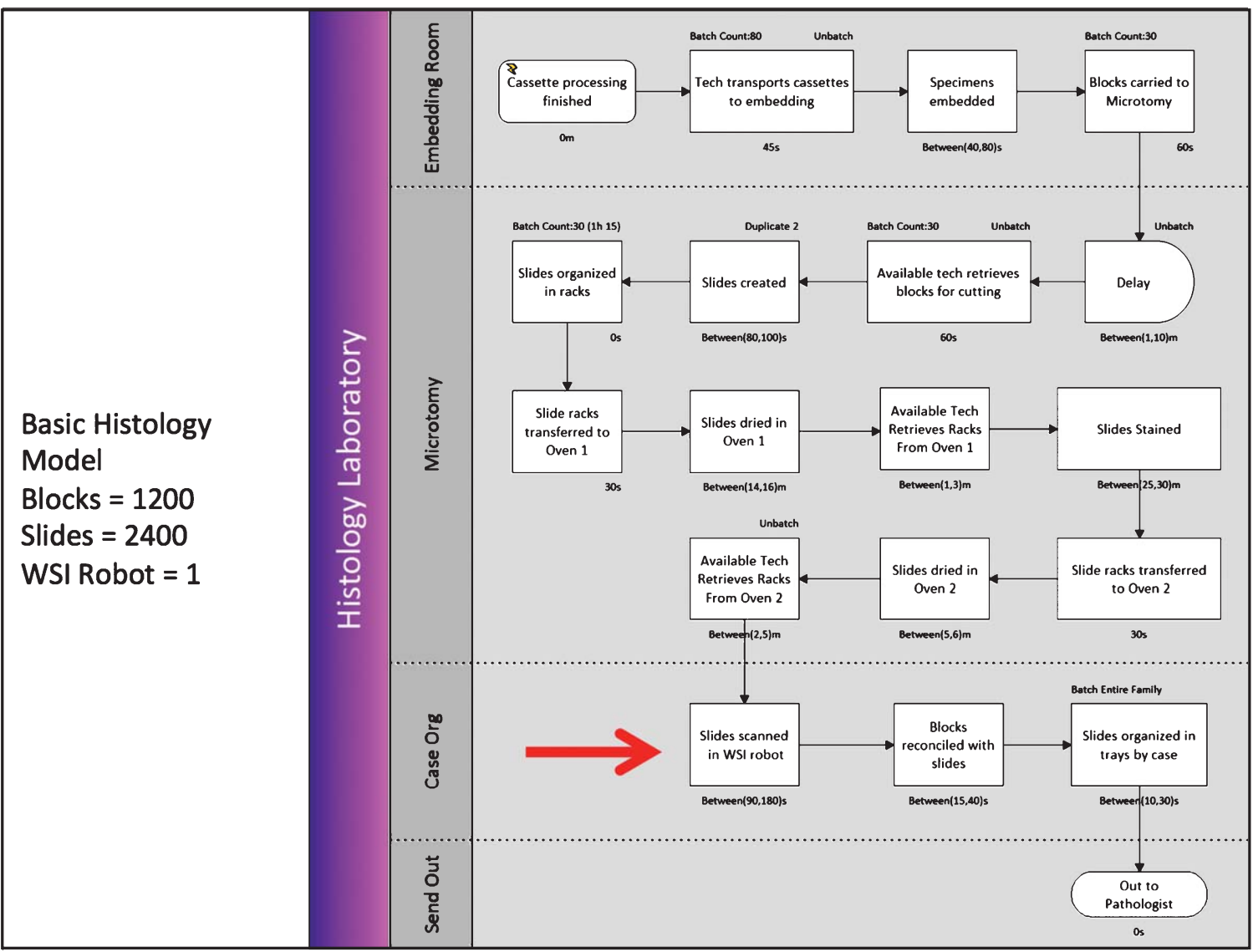

Fig. 4. WSI Robot Process Model in iGrafx ${ }^{\circledR}$ 2011. The arrow marks where the WSI robot was placed within the histology workflow. 
WSI SCAN TIME: $60-180 \mathrm{sec}$

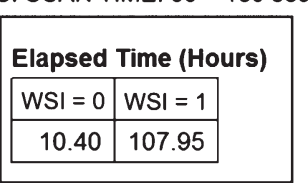

WSI SCAN TIME: $90-180 \mathrm{sec}$

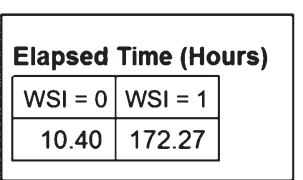

WSI SCAN TIME: $60-240 \mathrm{sec}$

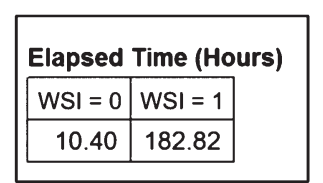

WSI SCAN TIME: $120-240 \mathrm{sec}$

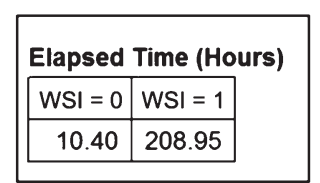

Fig. 5. Effects of a single WSI robot on turn-around-time (TAT) in a high-volume (2400 slides/day) clinical histology laboratory. The top rows in the above charts represent the process model used (baseline histology model: WSI $=0$, WSI robot process model: $\mathrm{WSI}=1)$. The bottom rows in the above charts represent the TAT (hours) needed to complete the simulation for each model.

To test this approach, a single WSI robot was placed within the histology laboratory process model and the scan times decreased in a step-wise manner. Simulations were run after each decrease in time and TAT measured until there was no difference between the baseline and WSI histology laboratory workflows. As seen in Fig. 7, with one WSI robot, scan times would have to decrease to $12-14 \mathrm{sec}$ per slide, a four to

five-fold decrease over the fastest scan times reported now $(60 \mathrm{~s})$ in order to have a minimal effect on the TAT for a high volume clinical histology laboratory.

\section{Conclusions}

Currently, the fastest WSI scan times quoted by most vendors are approximately $60 \mathrm{sec}$ per slide for high throughput systems; however, these scan times assume the most idealistic of scanning conditions and do not take into account errors that occur due to either the WSI robot or the glass slide itself. A major criticism of the simulations in this paper could be that there have been no accommodations made for rework within either the baseline histology process model or the WSI process model. Rework is commonly seen in histology laboratories - adding an additional step within the process can only increase the probability for additional rework. For the purposes of this initial study, we sought to test the effects of placing a WSI robot within a completely optimized, error-free system, both from a worker/equipment perspective and from a rework perspective. However, while this is a viable approach for isolating the effects of a single activity within a process, further work must be done

WSI SCAN TIME: $60-180 \mathrm{sec}$

\begin{tabular}{|c|c|c|c|c|c|c|c|c|c|c|c|c|}
\hline \multirow{3}{*}{$\begin{array}{l}\text { \# WSI Robots } \\
\text { Histology TAT }\end{array}$} & \multicolumn{12}{|c|}{ Elapsed Time (Hours) } \\
\hline & 0 & 1 & 2 & 3 & 4 & 5 & 6 & 7 & 8 & 9 & 10 & 11 \\
\hline & 10.40 & 107.95 & 54.60 & 34.64 & 27.92 & 17.39 & 14.69 & 12.78 & 11.34 & 10.45 & 10.42 & 10.42 \\
\hline
\end{tabular}

WSI SCAN TIME: $90-180 \mathrm{sec}$

\begin{tabular}{l|c|c|c|c|c|c|c|c|c|c|c|c|c|}
\hline \multicolumn{10}{|c|}{} & \multicolumn{10}{c|}{ Elapsed Time (Hours) } \\
\cline { 2 - 13 } & $\begin{array}{l}\text { \# WSI Robots } \\
\text { Histology TAT }\end{array}$ & 1 & 2 & 3 & 4 & 5 & 6 & 7 & 8 & 9 & 10 & 11 & 12 \\
\hline & 10.40 & 172.27 & 59.50 & 37.91 & 30.38 & 25.85 & 16.33 & 14.18 & 12.57 & 11.31 & 10.45 & 10.43 & 10.43 \\
\hline
\end{tabular}

WSI SCAN TIME: $60-240 \mathrm{sec}$

\begin{tabular}{l|c|c|c|c|c|c|c|c|c|c|c|c|c|c|}
\hline \multicolumn{10}{|c|}{} & \multicolumn{10}{|c|}{ Elapsed Time (Hours) } \\
\begin{tabular}{|c|c|c|c|c|c|c|c|c|c|c|} 
\# WSI Robots \\
Histology TAT
\end{tabular} & $\mathbf{1}$ & $\mathbf{2}$ & 3 & 4 & 5 & 6 & 7 & 8 & 9 & 10 & $\mathbf{1 1}$ & $\mathbf{1 2}$ & 13 \\
\hline 10.40 & 182.82 & 64.78 & 41.44 & 33.01 & 27.97 & 24.59 & 15.70 & 13.89 & 12.49 & 11.37 & 10.51 & 10.42 & 10.42 \\
\hline
\end{tabular}

WSI SCAN TIME: $120-240 \mathrm{sec}$

\begin{tabular}{|c|c|c|c|c|c|c|c|c|c|c|c|c|c|c|c|c|}
\hline \multirow{3}{*}{$\begin{array}{l}\text { \# WSI Robots } \\
\text { Histology TAT }\end{array}$} & \multicolumn{16}{|c|}{ Elapsed Time (Hours) } \\
\hline & 0 & 1 & 2 & 3 & 4 & 5 & 6 & 7 & 8 & 9 & 10 & 11 & 12 & 13 & 14 & 15 \\
\hline & 10.40 & 208.95 & 81.10 & 54.48 & 37.93 & 31.89 & 27.86 & 25.00 & 16.35 & 14.67 & 13.32 & 12.22 & 11.31 & 10.55 & 10.43 & 10.43 \\
\hline
\end{tabular}

Fig. 6. Number of WSI robots needed to maintain the current workflow in a high-volume (2400 slides/day) clinical histology laboratory. Bolded numbers in the above charts denote the point at which additional WSI robots have little to no effect on histology lab turn-around-time (TAT). 


\begin{tabular}{|c|c|c|c|c|c|c|c|c|c|} 
Slide scan time (sec) & $120 \mathrm{~s}$ & $60 \mathrm{~s}$ & $30 \mathrm{~s}$ & $20 \mathrm{~s}$ & $15 \mathrm{~s}$ & $\mathbf{1 4 s}$ & $\mathbf{1 3 s}$ & $\mathbf{1 2 s}$ & $10 \mathrm{~s}$ \\
\cline { 2 - 10 } Histology TAT (hours) & 107.22 & 54.22 & 27.72 & 14.56 & 11.23 & 10.58 & 10.41 & 10.40 & 10.40 \\
\cline { 2 - 10 }
\end{tabular}

Fig. 7. Average WSI robot scan times needed for a single robot to maintain the current workflow in a high-volume (2400 slides/day) clinical histology laboratory. Bolded numbers above denote the point at which further decreases in the slide scan time have little to no effect on histology lab turn-around-time (TAT).

in order to better model the reality of both the clinical histology laboratory and the whole slide imaging robot workflows.

While the results of this study show to a great extent that WSI, in its present form, is not ready for full adoption in high-volume clinical histology laboratories, the converse is also true - existing workflows and processes of clinical histology laboratories are not ready for whole slide imaging. It is unrealistic, at least at the present time, to expect that a WSI robot will be able to perfectly scan an entire glass slide in 14 seconds from start to finish. Nor is it realistic to expect pathology practices to purchase such large numbers of these devices given today's financial constraints within healthcare and space constraints within hospitals. Instead, it seems more likely that if WSI can provide enough value added to pathology [11], then the workflows and processes involved in delivering the glass slides to the WSI robot must first be evaluated and optimized in order for full adoption to proceed.

Whole slide imaging holds great promise in pathology as being both an innovative and disruptive technology. While great strides have been made in advancing this field technically, from a logistical standpoint there remains a long road ahead towards the full adoption of WSI within pathology. Through the use of business analysis software, process modeling, and computerized simulations, clear assessments using real laboratory data can be made in order to better understand current and future impacts of workflow and process changes.

\section{References}

[1] R.S. Weinstein, A.R. Graham, L.C. Richter, G.P. Barker, E.A. Krupinski, et al., Overview of telepathology, virtual microscopy, and whole slide imaging: Prospects for the future, Hum Pathol 40 (2009), 1057-1069.

[2] C.V. Hedvat, Digital Microscopy: Past, Present, and Future, Arch Pathol Lab Med 134 (2010), 1666-1670.

[3] L. Pantonowitz, P.N. Valenstein, A.J. Evans, K.J. Kaplan, J.D. Pfeifer, et al., J Pathol Inform 2 (2011), 36.

[4] J. Gilbertson and Y. Yagi, Histology, imaging, and new diagnostic work-flows in pathology, Diagn Pathol 15 (Suppl 1) (2008), S14.

[5] B.G. Sobolev, V. Sanchez and C. Vasilakis, Systematic review of the use of computer simulation modeling of patient flow in surgical care, J Med Syst 35 (2011), 1-16.

[6] G.R. Hung, S.R. Whitehouse, C. O’Neill, A.P. Gray and N. Kissoon, Computer modeling of patient flow in a pediatric emergency department using discrete event simulation, Pediatr Emerg Care 23 (2007), 5-10.

[7] R. Feyrer, J. Rösch, M. Weyand and U. Kunzmann, Costunit accounting based on a clinical pathway: A practical tool for DRG implementation, Thorac Cardiovasc Surg 53 (2005), 261-266.

[8] R. Feyrer, U. Kunzmann and M. Weyand, Computer-assisted process simulation: A suitable instrument for process optimization in hospitals, Zentralbl Chir 131 (2006), 347-353.

[9] G.J. Buffone, D. Moreau, and J.R. Beck, Workflow computing: Improving management and efficiency of pathology diagnostic services, Am J Clin Pathol 105 (4 Suppl 1) (1996), S17-S24.

[10] M.G. Rojo, E. Rolón, L. Calahorra, F.O. Garcia, R.P. Sánchez, et al., Implementation of the Business Process Modelling Notation (BPMN) in the modelling of anatomic pathology processes, Diagn Pathol 3 (Suppl 1) (2008), S22.

[11] M. Isaacs, J.K. Lennerz, S. Yates, W. Clermont, J. Rossi, and J.D. Pfeifer, Implementation of whole slide imaging in surgical pathology: A value added approach, J Pathol Inform 2 (2011), 39. 


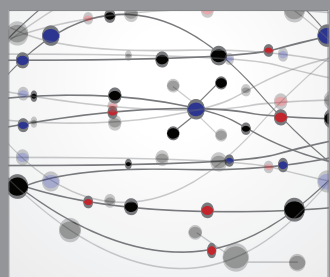

The Scientific World Journal
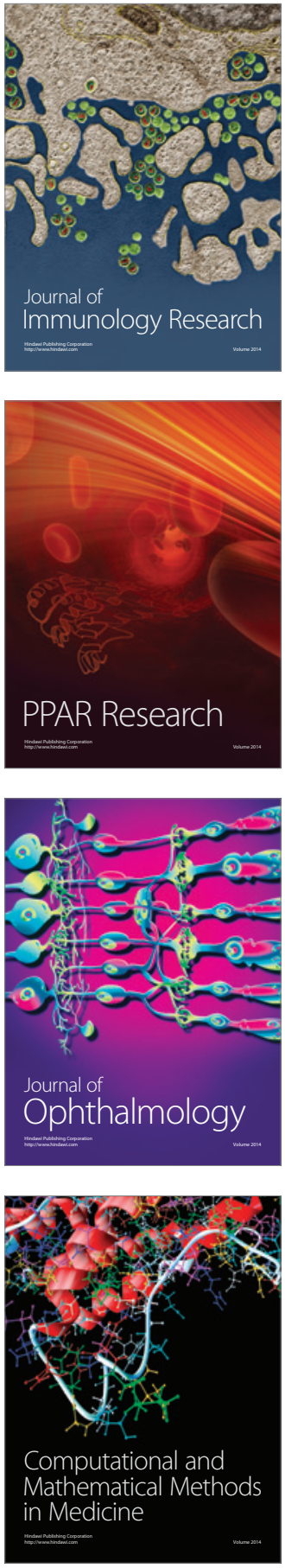

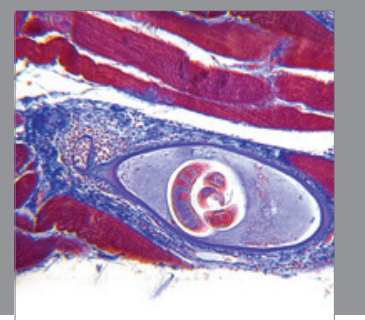

Gastroenterology

Research and Practice
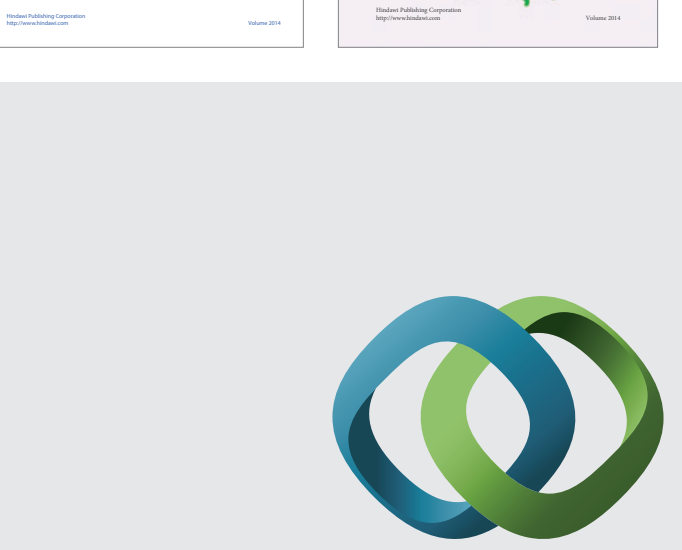

\section{Hindawi}

Submit your manuscripts at

http://www.hindawi.com
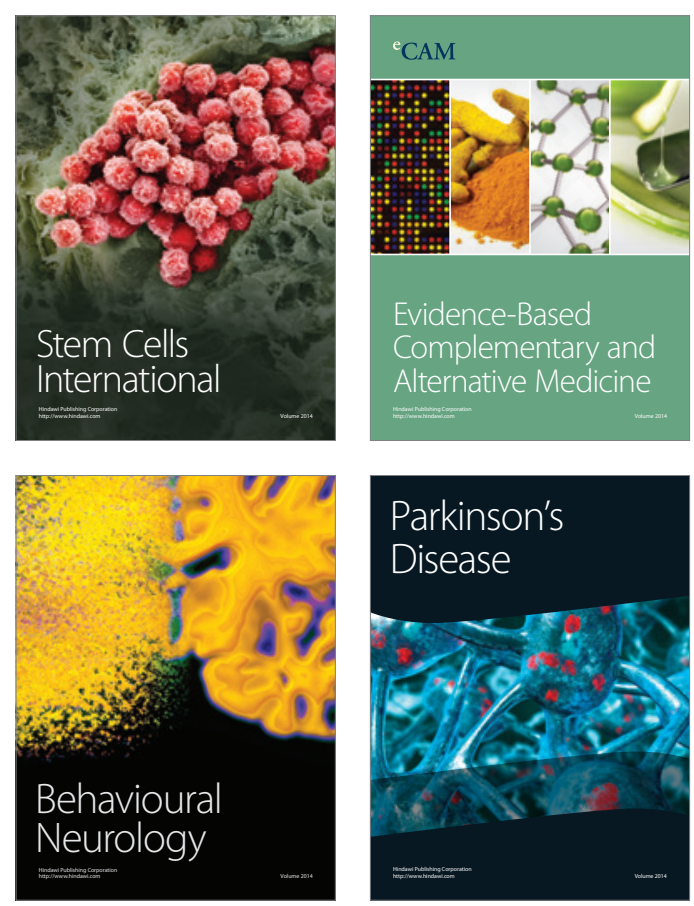

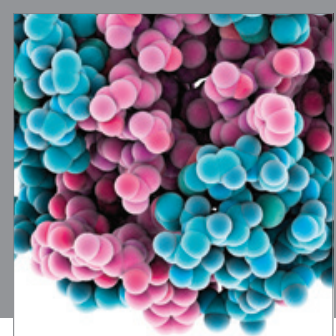

Journal of
Diabetes Research

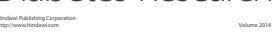

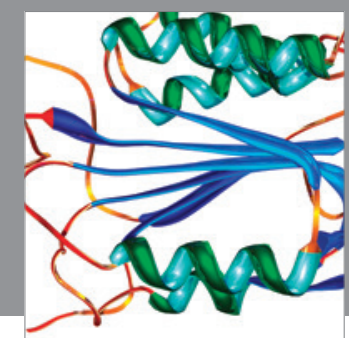

Disease Markers
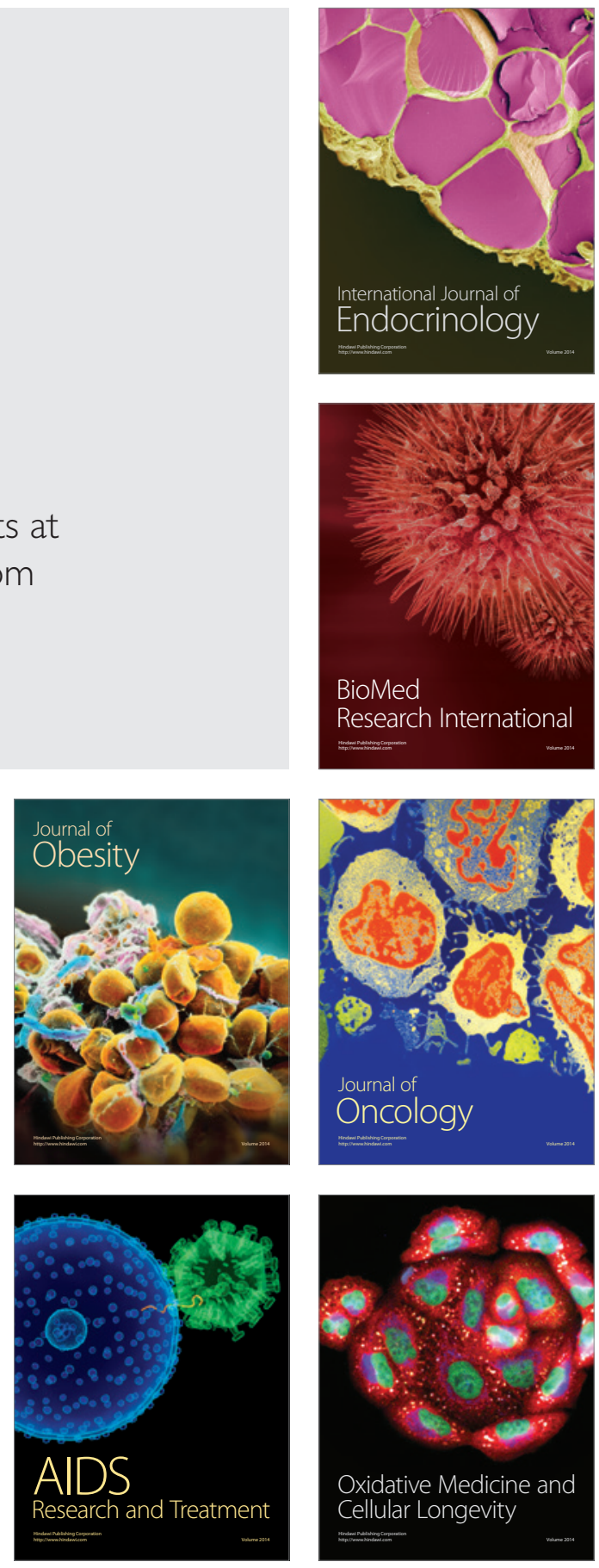